\title{
PROTECCIÓN DE LOS DERECHOS LABORALES PARA LOS REINSERTADOS EN EL CONTEXTO DE LA EMPRESA PRIVADA COLOMBIANA
}

\author{
Sandra Elena Anaya Sierra ${ }^{1}$ \\ Katia Palencia Sánchez
}

\section{Resumen}

El presente escrito desarrolla la caracterización del trabajo en condiciones dignas y justas, enmarcado en el Estado social de Derecho, con prevalencia del Derecho de igualdad, y el Derecho al trabajo, promovido mediante políticas públicas que contribuyan en el fomento del empleo, logrando que el ser humano- trabajador- se sienta útil en la sociedad al satisfacer sus necesidades básicas y las de su núcleo familiar. Visualizar el trabajo como Derecho Fundamental cuya protección está en cabeza del Estado, otorgando, entre otros, beneficios de protección a los menos favorecidos como lo son los Reinsertados, enfocándonos en la categoría de reinsertados que han cumplido penas privativas de la libertad y desean retomar una vida basada en la legalidad, pero en muchas ocasiones enfrenta la discriminación social fundamentada en la negación por parte del empleador cuando intentan ocupar un cargo. A lo anterior se suma la falta de intervención del Estado mediante políticas públicas eficientes y el seguimiento que debe hacerse que permitan garantizar la resocialización objetiva para que de esta manera se contribuya con la paz social y se frene la reincidencia del delito. Para esto se proponen unas herramientas que contribuirían lograr la protección de derechos laborales a estas personas. Lo anterior constituye un trabajo de tipo descriptivo, realizado bajo un enfoque cualitativo, empleando fuentes secundarias de información, teniendo como base fuentes documentales. A través de este escrito se logrará integrar la realidad jurídica laboral con el contexto social y dar un enfoque que genere o contribuya protección al Derecho al trabajo de las personas reinsertadas en Colombia.

Palabras Clave: Derecho, Estado social de derecho, trabajo, derecho fundamental, políticas públicas, reinsertado, constitución política, código laboral.

${ }_{1}^{1}$ Candidata a Magister, Especialista en Derecho Laboral y en Derecho Administrativo, Abogada. Docente de la Facultad de Derecho de la Corporación Universitaria del Caribe. Adscrita al Centro de investigación Sociojurídicas de la Corporación Universitaria del Caribe GISCER. Email: sandra.anaya@cecar.edu.co 


\title{
Sandra Elena Anaya Sierra
}

\begin{abstract}
This paper develops the characterization of work in decent and fair conditions, framed in the Social Rule of Law, with prevalence of the Equality Law, promoted through public policies that contribute to the promotion of employment, achieving that the worker-human being- feel useful in society by satisfying their basic needs and those of their family nucleus. Visualize the work as Fundamental Law whose protection is at the head of the State, granting, among others, protection benefits to the less favored ones such as the Reinserted, focusing on the category of reinserted who have served prison sentences and wish to resume a life based on legality, but often faces social discrimination based on denial by the employer when trying to hold a position. To this is added the lack of intervention by the State through efficient public policies and the follow-up that must be done to guarantee objective re-socialization so that social peace can be contributed in this way and the recidivism of crime can be stopped. For this purpose, some tools are proposed that would help to achieve the protection of labor rights for these people. This is a descriptive work, carried out under a qualitative approach, using secondary sources of information, based on documentary sources. Through this paper we will be able to integrate the labor legal reality with the social context and give an approach that generates or contributes protection to the Right to work of the reinserted people in Colombia.
\end{abstract}

Keywords: Law, Social state of law, labor, fundamental law, public policies, reinserted, political constitution, labor code.

\section{Introducción}

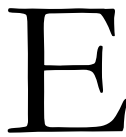

1 trabajo ha sido desde antaño un medio de subsistencia propio del ser humano, la palabra trabajo significa faena, labor, tarea, actividad involucra un despliegue físico de energía material o intelectual.

Desde el punto de vista etimológico la palabra trabajo viene de la voz latina tranbs-trabis que significa traba, obstáculo o dificultad para poder el hombre satisfacer sus mínimas necesidades. Igualmente se encuentra otra locución latina labor-laboris, que significa actividad encaminada a realizar algo, labor es sinónimo de actividad, ocupación (Lopez Cardenas Jairo Alfonso, 2015, pág. 20).

Desde el punto de vista jurídico trabajo es toda actividad humana, libre ya sea material o intelectual, permanente o transitoria, que una persona natural ejecuta consiente al servicio de otra persona natural o jurídica , cualquiera que sea su finalidad y en ejecución de un contrato de trabajo, 
no obstante ante la importancia social de trabajo, debe ser preocupación de todo gobierno fomentar las políticas efectivas de fomento y conservación del empleo, como lo es sentirse útil realizando la profesión u oficio que haya escogido de esta manera procura sus subsistencia y la de su núcleo familiar, colocando a disposición del empleador su fuerza de trabajo y recibiendo un salario justo.

En Colombia durante la época denominada auge del capitalismo industrial se da una gran demanda de mano de obra a medida que aumentaba el número de empresas y el poder industrial de ella, el abandono de los campos por los trabajadores, atraídos por la posibilidad de mejor salario y alagados por posibles comodidades materiales, van haciendo que aquella masa adquiera dimensiones gigantescas, aunque desordenadas al principio, tanto desde el punto de vista disciplinario y doctrinario, como por el desamparo y la indiferencia social (Gonzalez Charry Guillermo, 2016, pág. 10).

No obstante en la Constitución política de Colombia de 1991 fueron elevados a normas constitucionales y como derechos fundamentales varios de los principios que anteriormente eran meramente legales, es decir, vario de los postulados que trae el Código Sustantivo del Trabajo tanto en su parte individual y colectiva. La finalidad del Código Del Trabajo es otorgar al trabajador, que es la parte más débil de la relación laboral, una existencia digna y un nivel económico decoroso para él y su núcleo familiar (Lopez Cardena Jairo Alfonso, 2015, pág. 25).

En el siglo XX se demarca por el nacimiento y la consolidación de una disciplina jurídica laboral que regula las relaciones entre capital y trabajo.

En este escrito se propone identificar aspectos destacados de la legislación laboral dentro de un contexto jurídico vigente confrontado con una realidad social como lo es la Reinserción laboral en la empresa pri- vada, por lo tanto se hace necesario enfocar posibles soluciones ante la evidente exclusión laboral de estas personas que impiden su resocializa- ción e incluso caen en la reincidencia, para esta manera dar protección al trabajo desarrollado en un contexto digno y justo en busca del bien, la paz y la armonía social. 


\section{Metodología}

Para el desarrollo de la investigación se planteó un diseño metodológico estructurado en el paradigma de la investigación cualitativo, apoyado en el enfoque hermenéutico, e implementando en momentos justificados los tipos de investigación exploratorio, descriptivo y correlacional, de acuerdo a la estrategia trazada. Empleando fuentes secundarias de información, teniendo como base fuentes documentales, manifiestas en fuentes formales del Derecho laboral y en balances y producciones sobre componentes científicos del mismo en Colombia.

Como indica Palencia (Palencia, 2009) el enfoque es cualitativo, puesto que la investigación se orienta a abordar el objeto de estudio a partir de las cualidades que lo definen y caracterizan, desde los hechos, procesos y estructuras. Todo a partir de una revisión literaria constante y un proceso de hermenéutico de las instituciones y seres vivos relacionados con el tema de estudio. Las técnicas de información son la lectura del material bibliográfico y análisis de la información.

\section{Protección de los derechos laborales para los reinsertados en el con- texto de la empresa privada}

El Derecho al trabajo es la base para gozar de una vida digna. Toda persona debería tener la posibilidad de un trabajo decente. Visualizar el trabajo como una condición básica y fundamental a lo largo de toda la vida debe estar enmarcado dentro de las relaciones laborales que guarden el respeto y la dignidad humana en el contexto legal.

La dignidad humana es un principio fundante del ordenamiento jurídico y del Estado colombiano que constituye un derecho fundamental autónomo. Además, pasa a ser valorado como sujeto individual y social teniendo en cuenta la igualdad de circunstancias basados en el respeto.

Por venir de la especie humana somos merecedores de diferentes derechos como el derecho a la vida, a la educación, al trabajo, a la igualdad, entre otros, con la única condición de guardar el respeto con los demás, entonces al momento de ejecutar actos que atenten contra vínculo laborales como maltratar, humillar, discriminar, excluir etc., estaríamos afectando la dignidad humana. 
El derecho al trabajo es un derecho fundamental de todas las personas y está garantizado en la Constitución Política Colombiana, es decir, que este derecho nace con el ser humano. En la Constitución Política el trabajo representa un valor esencial que se erige en pilar fundamental del Estado Social de Derecho, en cuanto lo reconoce como un Derecho en cabeza de toda persona a pretender y obtener un trabajo en condiciones dignas y justas, e igualmente como una obligación social, fundada en la solidaridad social (Guillermo Guerrero Figueroa, 2016, pág. 63).

En Colombia el trabajo es un Derecho y una obligación social, el Estado es garante de este derecho fundamental al ser el encargado de dar protección al trabajo en condiciones dignas y justas a través de los funcionarios públicos y mediante las políticas públicas que contribuyan al fomento y la protección del empleo.

La Constitución Política desde su preámbulo tiene como fundamento, basado en la declaración de los propósitos de las normas en con- cordancia con los Fines Esenciales del Estado, consagra derechos, deberes y formulaciones, asegura a sus integrantes, el Trabajo digno basado en la obligatoriedad, entre otros aspectos.

El Artículo Primero de la carta magna entre sus apartes consagra el respeto a la dignidad humana como aspecto importante en un EstadoSocial de Derecho, el artículo segundo consagra los Fines Esenciales del estado lo busca siempre la protección legal de los coasociados.

Artículo 1. (Asamblea Nacional Constituyente, 1991, pág. 1) Colombia es un Estado social de derecho, organizado en forma de República unitaria, descentralizada, con autonomía de sus entidades territoriales, democrática, participativa y pluralista, fundada en el respeto de la dignidad hu- mana, en el trabajo y la solidaridad de las personas que la integran y en la prevalencia del interés general.

Artículo 2. (Asamblea Nacional Constituyente, 1991, pág. 1) Son fines esenciales del Estado: ser- vir a la comunidad, promover la prosperidad general y garantizar la efectividad de los principios, derechos y deberes consagrados 
en la Constitución; facilitar la participación de todos en las decisiones que los afectan y en la vida económica, política, administrativa y cultural de la Nación; defender la independencia nacional, mantener la integridad territorial y asegurar la convivencia pacífica y la vigencia de un orden justo. Las autoridades de la República están instituidas para proteger a todas las personas residentes en Colombia, en su vida, honra, bienes, creencias, y demás derechos y libertades, y para asegurar el cumplimiento de los deberes sociales del Estado y de los particulares.

El artículo 13 de la carta magna, establece el Derecho de igualdad y busca evitar la discriminación:

Artículo 13. (1991, 1991, pág. 1). Todas las personas nacen libres e iguales ante la ley, recibirán la misma protección y trato de las autoridades y gozarán de los mismos derechos, libertades y oportunidades sin ninguna discriminación por razones de sexo, raza, origen nacional o familiar, lengua, religión, opinión política o filosófica. El Estado promoverá las condiciones para que la igualdad sea real y efectiva y adoptara medidas en favor de grupos discriminados o marginados. El Estado protegerá especialmente a aquellas personas que, por su condición económica, física o mental, se encuentren en circunstancia de debilidad manifiesta y sancionará los abusos o maltratos que contra ellas se cometan.

En Colombia el trabajo es un derecho y una obligación social, consagrada en el artículo 25 de la C.P preceptúa "El trabajo es un derecho y una obli- gación social y goza, en todas sus modalidades, de la especial protección del Estado. Toda persona tiene derecho a un trabajo en condiciones dignas y justas". La expresión en condiciones dignas y justas quiere decir que nin- gún trabajador puede desarrollarse en ambientes vejatorios para la digni- dad humana o nocivos para la vida, todo vínculo laboral debe ser respe- tuoso de la dignidad del trabajador sin admitir situaciones de hecho o prescripciones legales o contractuales que por su carácter absurdos o leo- nino, o por provocar algún desmedro físico o moral, atente contra la calidad humana del trabajador (Guillermo Guerrero Figueroa, 2016). 
El Estado es garante de este derecho fundamental, es el encargado de dar protección al trabajo en condiciones dignas y justas mediante los funcionarios públicos y mediante las políticas públicas que contribuyan al fomento y la protección del empleo entre otras.

La Constitución Política en su Artículo 53, Contempla los mínimos de derechos y garantías consagrados a favor de los trabajadores, los cuales detallo así:

- Igualdad de oportunidades para los trabajadores

- Remuneración mínima vital y móvil, proporcional a la cantidad y calidad de trabajo;

- Estabilidad en el empleo

- Irrenunciabilidad a los beneficios mínimos establecidos en normas laborales

- Facultades para transigir y conciliar sobre derechos inciertos y discutibles;

- Situación más favorable al trabajador en caso de duda en la aplicación e interpretación de las fuentes formales de derecho

- Primacía de la realidad sobre formalidades establecidas por los sujetos de las relaciones laborales

- Garantía a la seguridad social, la capacitación, el adiestramiento y el descanso necesario;

- Protección especial a la mujer, a la maternidad y al trabajador menor de edad.

- El Estado garantiza el derecho al pago oportuno y al reajuste periódico de las pensiones legales.

- Los convenios internacionales del trabajo debidamente ratificados, hacen parte de la legislación interna. La ley, los contratos, los acuerdos y convenios de trabajo, no pueden menoscabar la libertad, la dignidad humana ni los derechos de los trabajadores.

La protección legal contemplada en el ordenamiento jurídico vigente no solo está plasmada en la Constitución Política como normas de normas, también se contempla la protección legal en normas plasmadas en el Código Laboral colombiano, las leyes que lo modifican o adicionan en los 
convenios de la O.I.T. ratificados por el congreso colombiano, la jurisprudencia y la doctrina. Toda esta protección legal en aras de preservar el trabajo digno, aunque muchas veces no se logra por malas prácticas de algunos empleadores o malas asesorías jurídicas.

Dentro de la protección legal consagrada en el Código Sustantivo del Trabajo podemos citar lo preceptuado en el artículo 11, dice: "Toda persona tiene derecho al trabajo y goza de libertad para escoger profesión u oficio, dentro de las normas prescritas por la constitución y la Ley"

El artículo 9- "El trabajo goza de la protección del Estado, en la forma prevista en la Constitución Nacional y las leyes. Los funcionarios públicos están obligados a prestar a los trabajadores una debida y oportuna protección para la garantía y eficiencia de sus derechos, de acuerdo con sus atribuciones.

El artículo 11 del Código Sustantivo del trabajo dice: “Toda persona tiene derecho al trabajo y goza de libertad para escoger profesión u oficio, dentro de las normas prescritas por la Constitución y la Ley"

Los convenios y tratados de la organización internacional del trabajo rati- ficados por el congreso colombiano tales como: C 019 convenio sobre la igualdad de trato, C100 Convenio sobre igualdad de remuneración, C111 Convenio sobre la discriminación, C081 Convenio sobre la inspección de trabajo, C002 Convenio sobre el empleo, entre otros convenios ratificados mediante los cuales el Estado ha tratado de dar garantías a sus coasociados.

Como podemos evidenciar existe un gran espectro jurídico que con lleva a la protección del Derecho al trabajo en condiciones dignas y justas, no obstante, concebimos la reinserción laboral como parte integrante de un proceso de resocialización del individuo - trabajador que consiste en un proceso en el cual una persona que perdió su trabajo consigue uno nuevo- permitiéndole volver a ingresar al universo de trabajadores activos dentro del contexto de oferta y demanda del mercado laboral, la persona que pierde su empleo necesita vincularse rápidamente a un trabajo para devengar un salario y poder satisfacer sus necesidades y las de su núcleo familiar. Es el Estado el encargado de fomentar las políticas públicas que 
conlleven a la protección del trabajo en condiciones dignas y justas en aspectos como son formación de los trabajadores para la reinserción social, la entrega de subsidios y la dinamización de la economía en general, etc.

Una persona que cometió un delito y es condenada penalmente a una pena privativa de la libertad por un tiempo prolongado y por esta situación se genera una causal de terminación de su contrato de trabajo, en pocas palabras, pierde su empleo, necesitarían esta persona que el Estado le brinde mediante una política pública una ayuda que conlleve a resocializarlo para que en el momento de salir de la cárcel no reincida en el delito, aquí entraría la reinserción laboral a Construirse desde la misma cárcel, enseñándolo a visualizar una nueva vida laboral basado en el trabajo digno que conllevaría a lograr su propia subsistencia y la de su núcleo familiar una vez quede en libertad.

La reinserción laboral, en el caso antes anotado, forma parte importante de la reinserción social de un individuo que estuvo en una larga espera de su libertad y quien desea y está preparado para servirle a la sociedad formando parte de una comunidad y dentro de la legalidad para que jamás reincidan o incursionen en conducta delictiva.

$\mathrm{Al}$ momento de intentar ocupar un cargo en una empresa privada, el reinsertado podría encontrar las siguientes situaciones que obstaculizan la resocialización laboral en una empresa como podrían ser:

- Intolerancia y estigmatización por haber cometido una conducta criminal con las respectivas anotaciones en su certificado de antecedentes judiciales, aunque haya pagado la correspondiente pena, y como consecuencia de esto se evita a toda costa una contratación laboral

- Los que creen que estas personas tienen derecho a una segunda oportunidad y se la otorgan, bajo la convicción de que pueden convertirse en personas de bien.

- Los que creen que estas personas tienen derecho a una segunda oportunidad, pero evitan la contratación laboral, aludiendo motivos distintos, pero en realidad se enfoca más en la desconfianza que le generan éstas. 
No obstante, la falta de confianza en uno mismo es quizás el peor enemigo a la hora de buscar trabajo.

Los planes de reinserción laboral pretenden demostrar a los participantes que tienen más herramientas de las que notan, y que siempre pueden formarse y apuntar más alto (Julian Perez y Ana Gardey, 2015).

"Los presos que intentan reintegrarse en la sociedad se encuentran con diversas barreras debido a su pasado penitenciario", argumentó Augusto Rueda, jefe del Ministerio Público de Bucaramanga. También destacó que eliminar señalamientos y juicios es el primer paso para permitir la reinserción social de los sentenciados.

"Pidieron mi cédula y una vez la registraron se enteraron que estuve recluido, solo me dijeron que la empresa tenía prohibido entregar productos a personas en mi condición", comentó uno de los afectados (Redaccion el Tiempo, 2015).

Toda esta armadura jurídica garantista y proteccionista sumada a la intervención del Estado mediante las políticas públicas de resocialización del individuo desde la cárcel, tratando de fomentar el hábito del trabajo digno, de enseñarle un oficio, logra prepararlo para enfrentarse a un entorno laboral exigente con fin de que no caiga en la reincidencia del delito y se convierta en su ser útil a la sociedad, pero denotamos que choca esta política con la falta de oportunidades laborales para estas personas que buscar reinsertarse a la sociedad de una manera legal.

Se evidencia que para el sector privado los reinsertados no son personas atractivas para cumplir un rol laboral en sus empresas, generan para el empleador cierto impresión de riesgo de falta de confiabilidad, muy pocos empleadores son conscientes de lo valioso que es darle a una persona reinsertada una oportunidad laboral mediante un trabajo digno.

Ante todo lo anterior surge un gran interrogante: ¿En el caso concreto de los reinsertados el Estado colombiano cumple con dar protección al trabajo en condiciones dignas y justas enmarcadas en el Derecho de Igualdad dentro de un contexto del Estado Social de Derecho?

Antes de dar respuesta al interrogante, preciso lo siguiente: El Estado Social de Derecho busca entre sus apartes y de manera especial el equilibrio social, no obstante, y desafortunadamente se está percibiendo que 
se da un sentido meramente formal, pareciéndose cada día más al Estado de Derecho que estuvo vigente antes de la constitución política de 1991, atendiendo exclusivamente a un concepto formal de igualdad y libertad por cuanto se violan o ignoran los preceptos contemplados en la Carta Magna, encontrándonos los coasociados en desventaja en cuanto a la protección de sus derechos, deberes y prerrogativas, especialmente denotando desprotección a los derechos fundamentales, causando un desequilibrio social y afectando la vida digna, el empleo, la supervivencia y la convivencia pacífica.

En el interrogante que nos ocupa considero que el Estado ha sido ineficiente con relación a esta problemática de reinserción laboral de estas personas en la protección de su derecho al trabajo en condiciones dignas y justas. El Estado está fallando por cuanto no ha ajustado sus políticas públicas existentes en el contexto de la realidad social que vivimos donde prima el rechazo laboral y la estigma a estos seres humanos, donde prima una realidad dolorosa por falta de oportunidad laboral lo cual lleva a la reincidencia del delito al negarles una segunda oportunidad de cambio favorable, donde lo más probable es que logre edificarse a nivel personal siendo sujeto de otros derechos como podría ser el derecho a un salario mínimo vital y móvil entre otros.

El trabajo le permite al ser humano crecer como individuo dentro de la sociedad a la cual puede aportarle en la medida de sus posibilidades.

El Estado puede mejorar esta situación de la siguiente manera:

- Promover y concertar con el sector privado una política de reinserción laboral que consista en convocatorias de empleo con una etapa de selección y contratación laboral seria y objetiva, atendiendo a los buenos postulados en Trabajo Digno dentro del marco jurídico vigente (Derecho al olvido y perdón), que conlleven a evitar la discriminación laboral y a desdibujar el estigma de ex presidiario colocando en práctica el principio del respeto haciendo el reconocimiento del Derecho ajeno para lograr una sociedad en paz y en armonía social siendo tolerantes. 
- Lograr la inclusión de estas personas en lo que se ha denominado Estabilidad Laboral Reforzada por tener derecho al acceso y conservación de un trabajo decente y por haber pasado por una situación de privación de libertad.

- Invertir recursos en promoción a la no discriminación laboral a las personas reinsertadas en el sector privado mediante el Minis- terio de Trabajo y el Ministerio de Justicia con el fin de ejecutar una acción preventiva en la violación al Derecho al trabajo en condiciones dignas y justas y propender a la protección del derecho de igualdad y a la aplicación de los mínimos de derechos y garantías consagrados a favor de los trabajadores y a su vez evitar la exclusión social que se presenta cuando intentan acceder al mercado laboral. Tratar de mostrarle al empleador privado que es importante tenerlos encuentra vinculándolos a su entorno laboral para contribuir en la resocialización para evitar una posible recaída en el delito y así ayudarlos a la recuperación de su autoestima, a prestar sus servicios laborales, a sentirse útil en su trabajo, en la familia, en sociedad, a conseguir nuevas amistades, a generar su propia economía, ser autosuficiente, dignos de respeto y de admiración por su resocialización.

La labor de resocialización en el caso de los reinsertados es solo del Estado por cuanto el mismo Estado y las instituciones políticas y jurídicas que se fundan en su estructura teniendo por objeto y razón de ser a la persona, tales principios son extensivos a las relaciones laborales entre particulares quienes también están sujetos a la Constitución y obligados acatar sus prin- cipios.

Contribuir en la vinculación laboral de un reinsertado en una empresa del sector privado, mediante los modelos de empleabilidad generaría no solo beneficios en descuentos de impuestos para estas sino, que contribuiría a crear vínculos laborales de forma duradera basados en el respeto, el agradecimiento y el sentido de pertenencia, formar en ellos habilidades, valores y competencias, generaría estabilidad económica para estos, estas empresas son reconocidas por su gran aporte en la construcción de la paz 
y la armonía en un Estado social de Derecho, podría vincularlos como aprendiz SENA o por contratación directa.

\section{Conclusiones}

De lo anterior se puede concluir que debe existir una voluntad empresarial consistente en colaborarles a estas personas dándoles oportunidades labo- rales y capacitación, para cual la empresa debe tener contar con sus recur- sos económicos. También es necesario la identificación de las necesidades de la empresas y las posibles vacantes, los perfiles de los cargos a ocupar, la cultura organizacional de la empresa es determinante en este caso, la comunicación y acompañamiento de las personas asignadas para el pro- ceso de vinculación, enmarcado en el trato igual, no se admite trato dis- criminatorio, pero si debe existir un seguimiento en las labores realizadas en cuanto a su desempeño laboral que enfocaría el sentido de responsabi- lidad social y personal, trabajo en equipo, capacidad de atención, capacidad de saber escuchar, autonomía, dinamismo y cumplimiento de funciones a cabalidad, de otro lado sería importante que el empleador socializara in- terna y externamente la experiencia de vinculación de estas persona a su empresa convirtiéndose en ejemplo a seguir y contribuyendo con la paz del país.

De otro lado se considera que el Estado debe intervenir, como se planteó anteriormente en el fomento y seguimiento de nuevas políticas públicas que contribuyan con el fomento y conservación de empleo para estas personas, quienes por obvias razones se encuentran en desventaja en cuanto al ingreso laboral, también debe el Estado llevar unas estadísticas claras que arrojen la información de cuantas personal anualmente son reinsertadas laboralmente en la empresa privada, Ministerio de Trabajo.

El Derecho al trabajo pertenece al grupo de los Derechos económicos, sociales y culturales. El estado colombiano debe garantizar el Derecho al trabajo y de igualdad en el contexto laboral, no obstante no existe ningún decreto o ley cuya finalidad sea que la empresa privada de acuerdo con cierta cantidad de trabajadores pueda vincular en minoría a persona que hayan estado privado dela libertad. 


\section{Referencias}

Asamblea Nacional Constituyente. Constitucion Politica de Colombia (1991). Bogota D.C .

Asociación Colombiana de Facultades de Derecho. (2018). Acofade 15 años al Servicios de la Formación en Derecho. Bogota: Acofade.

Ausebel, D., Novak, J., \& Hanesian, H. (1983). Psicologia educativa.un punto de vista cognoscitivo. México: Trías Ed.

Bayuelo, P. (24 de abril de 2018). Acofade cumple 15 años de servicio a la comunidad juridica. Ambito Juridico. Recuperado el 13 de mayo de 2018, de https://www.ambitojuridico.com/noticias/academia/educacion-ycultura/acofade-cumple-15-anos-de-servicio-la-comunidad-juridica

Beltrán, M. (s.f). La importancia de la educación en los derechos bumanos. Especial referencia a América Latina. Obtenido de http://www.corteidh.or.cr/tablas/r24457.pdf

Carreño, Molina, \& Montoya. (2014). Componente Teórico y metodológico. En C. Molina , M. Carreño, R. Sayas, C. Montoya, \& A. M. Justicia (Ed.), Diagnóstico y lineamientos técnicos para los distintos escenarios de la práctica jurídica de los programas de derecho de las instituciones de educación superior en Colombia. (págs. 2144). Medellín: UniRemigton.

CECAR. (27 de Abril de 2018). Perfil ocupacional. Obtenido de Derecho: https://www.cecar.edu.co/programas/extensionsincelejo/pregrado/programas-modalidad-presencial/derecho.html

Clavijo, D. (2015). El enfoque de competencias en la formación del abogado del Siglo XXI. Justicia, 185-212.

Consejo nacional de acreditación de Colombia. (15 de marzo de 2018). Boletín estadistico 2016. Obtenido de https://www.cna.gov.co/1741/articles322100_Boletin_2016_def.pdf

Corporación Excelencia en la Justicia. (2008 - 2009). Reporte de justicia en las Américas. . Obtenido de Tasa de abogados por habitantes en Colombia y el mundo: http://www.cej. org.co/index.php/todos-los-justiciometros/2586tasa-de-abogados-por-habit

Corporación universitaria Antonio José de Sucre. (23 de marzo de 2018). Derecho. Obtenido de Corposucre:

http://web.corposucre.edu.co/programas/derecho

Delgado Ana Maria y Oliver rafael . (2003). Enseñanza de Derecho y tecnologias.

Obtenido de http//www.uoc.edu/dt/20310/index.html 
Dinero. (25 de Mayo de 2017). Obtenido de http://www.dinero.com/edicionimpresa/caratula/articulo/mejores-universidades-de-colombia-en-derechoen-2017/245822

Gadamer, H. (2005). Verdady Método. . Salamanca: Sígueme.

Gonzalez Charry Guillermo. (2016). Derecho Laboral Individual. Medellin.

Guillermo Guerrero Figueroa. (2016). Principios

Fundamentales Del Derecho AlTr Abajo. Bogota: LEYER.

Hernandez , R., Baptista , P., \& Collado , C. (2006). Metodología de la investigación. México: Mc Graw Hill.

Hernández, S. (2017). Breve diagnóstico sobre la oferta académica para la formación de los profesionales del Derecho. Revista de educación superior, 46(181), 55-74. doi:org/10.1016/j.resu.2016.10.003.

Jaimes, M. (2017). La educación no formal en Derechos Humanos. En P. Guzmán, Tendencias nacionales e internacionales en la formación de abogados (págs. 203-219). Barranquilla, Colombia: Universidad Simón Bolivar.

Julian Perez y Ana Gardey. (2015). Definicion de reinsercion laborañ. Bogotá.

Lopez Cardena Jairo Alfonso. (2015). Derecho Laboral Individual. Bogotà: Grupo Editorial Ibañez.

Lopez Cardenas Jairo Alfonso. (2015). Derecho Laboral Individual. Bogotà: Grupo editorial Ibañez.

Magendzo, A. (2006). Los derechos bumanos un objetivo transversal del currículum. . Obtenido dehttps://es.scribd.com/document/299437227/Los-DDHH-unObjetivo-Transversal-en-El-Curriculum-Abraham-Magendzo

Ministerio de educación. (26 de marzo de 2018d). Observatorio Laboral. Obtenido de http://bi.mineducacion.gov.co:8380/eportal/web/men-observatoriolaboral/instituciones-de-educacion-superiories1?p_auth=GOccZG5u\&p_p_id=com_ideasoft_o3_portlets_O3Control Portlet_WAR_o3portal_INSTANCE_P9ct\&p_p_lifecycle $=1 \&$ p_p_state= $n$ ormal\&p_p_mode $=$ view\&p_p

Ministerio de educación nacional. (4 de marzo de 2018a). Fichas técnicas, indicadores de eduación superior. Obtenido de https://www.mineducacion.gov.co/sistemasinfo/Informes/212350:Resume n-de-indicadores-de-Educacion-Superior

Ministerio de educación nacional. (14 de marzo de 2018b). Información nacional 2010-2016. Educación superior. Obtenido de https://www.mineducacion.gov.co/sistemasinfo/snies/ 
Ministerio de educación nacional. (26 de marzo de 2018c). Observatorio laboral. Obtenido de http://bi.mineducacion.gov.co:8380/eportal/web/menobservatorio-laboral/programas-

carreras1?p_auth=GOccZG5u\&p_p_id=com_ideasoft_o3_portlets_O3Co ntrolPortlet_WAR_o3portal_INSTANCE_cE3M\&p_p_lifecycle=1\&p_P_s tate $=$ normal\&p_p_mode $=$ view\&p_p_col_id=_118_INSTANCE

Ministerio de educación nacional. (14 de marzo de 2018e). SNIES módulo consultas. Obtenido de https://snies.mineducacion.gov.co/consu ltasnies/programa\#

Ministerio de educación nacional. República de Colombia. (01 de 05 de 2018). Resolución número 2768 del 13 de noviembre de 2003. Obtenido de https://www.mineducacion.gov.co/1621/article-86421.html

Ministerio de Justicia. (13 de abril de 2016). En Colombia, tres de cada 10 abogados se forman en programas académicos con acreditación de alta calidad. Recuperado de: Obtenido de http://www.minjusticia.gov.co/Noticias/TabId/157/ArtMID/1271/Articl eID/2296/En-Colombia-tres-de-cada-10-abogados-se-forman-enprogramas-acad233micos-con-acreditaci243n-de-alta-calidad.aspx

Molina, C. (12 de Enero de 2017). Nace el Colegio Profesional de Abogados de Colombia. Ámbito Jurídico, págs. 1-5.

Molina, C. M. (2017). Lineamientos técnicos para los programas de Derecho en Colombia. Medellin, Colombia: Fondo Editorial Corporación Universitaria Remington.

Molina, C., Carreño, M., Sayas, R., \& Montoya, C. (2014). Diagnóstico y lineamientos técnicos para los distintos escenarios de la práctica jurídica de los programas de derecho de las instituciones de educación superior en Colombia. (A. M. derecho, Ed.) Medellín: Uniremington.

Narváez, B. (2017). Pérfil de formación del abogado. Una mirada desde la formación en investigación del estudiante de Derecho. En P. Guzmán, Tendencias nacionales e internacionales en la formación de abogados (págs. 65-70). Barranquilla, Colombia: Ediciones Universidad Simón Bolivar.

Osorio, V. (2017). Practicas de los abogados en formación en los consultorios jurídicos. Revista Virtual Via Inveniendi et Iudicandi, 12(1), 119-148. doi:10.15332/s1909-0528.2017.0001.02

Palencia, M. (2009). Metodologia de la Investigación. Bogotá: UNAD.

Red Colsi. (2018). Obtenido de http://redcolsi.org/web/index.php?lang=en Red sociojuridica. (2018). Obtenido de https:/ / www.redsociojuridica.org/red/acerca-de/universidades-asociadas / 
Redaccion el Tiempo. (03 de Febrero de 2015). Redaccion el Tiempo.

Semana. (2 de Junio de 2017). Obtenido de http://www.semana.com/educacion/ articulo/universidad-de-los-andes-mejor-universidad-en-derecho/514295

Sistema nacional de información de la educación superior. (17 de marzo de 2018). SNIES- Módulo Consultas. Obtenido de https://snies.mineducacion.gov.co/consultasnies/programa

Universidad de Sucre. (27 de abril de 2018). Derecho. Obtenido de Facultad de educación y ciencias: http://www.unisucre.edu.co/educacion/index.php/programas/pregrado/d erecho

Vasquez. (2017). Modelos, corrientes y actores del Derecho en Colombia. Medellín: UNAULA. 